\title{
ONCHOCERCIASIS AND LEPROSY
}

by S. G. BROWNE, M.D., F.R.C.S., M.R.C.P., D.T.M.

Formerly in charge of Yalisombo Leprosarium, Belgian Congo, now of the Leprosy Research Unit, Uzuakoli, Eastern Nigeria.

(Reprinted article by kind permission, from "La Gale Filarienne et la Lèpre”, Ann. Soc. Belg. de Méd. Trop. 30 June, 1959, 39, 3: pp. 257-265. This English version of the original French has been approved by the author.)

The observations of Rodhain (1915) and of Dubois (1917) on the possible role of Onchocerca volvulus in the etiology of a chronic dermatitis in the Belgian Congo draw attention to the problem of "craw-craw" in other African territories (O’Neill, 1875, etc.). Since then many clinical and pathological enquiries were carried out by many doctors, from Ouzilleau (1921) to Van der Berghe (1941), and by others also.

These authors emphasised the cutaneous changes brought about by chronic onchocercal dermatitis. Considerable areas of skin are covered with excoriations, lichenifications, and pachydermia. It is true that some authors mention the pigmentary anomalies associated with a long-standing pruritis without making much of them, but the diagnostic difficulties which launched the present studies were not of this nature (Tisseuil, 1949).

The different elements which intermingle in the typical composite picture of onchodermatitis are usually easily recognizable and the different cutaneous forms of leprosy in most cases give no difficulty to the leprologist who is alert and experienced. But the real cutaneous resemblances between the two diseases have not received, in my opinion, the attention which their importance deserves (Browne, 1959).

It has recently been brought to my notice in the Belgian Congo that in many instances practical difficulties do indeed occur, especially where the two diseases exist together and where the percentage of persons attacked by each is quite high. For example, in a certain leprosarium about a third of so-called lepromatous, who had been bacteriologically negative for a long time, had to be discharged because they were not suffering from leprosy but from onchocerciasis. Elsewhere it is reported that nodular lepromatous cases exist which show no clinical improvement at the end of long periods of sulphone treatment: the "nodules" on the chest remain unchanged because they contain the adult filariae; i.e., they are onchocercomata.

It goes without saying that the presence of the microfilariae of Onchocerca volvulus and the absence of $M$. leprae do not mean that the lesion in question is certainly of onchocercal origin; and, on 
the other hand, that the presence of $M$. leprac and the absence of dermal microfilariae in a vague hypopigmented macule require the diagnosis of leprosy to the exclusion of onchocerciasis with regard to the lesion.

The present study, based on some 131 cases, tries to bring out the diagnosis difficulties from both points of view by underlining the deceptive appearances of filarial dermatosis in Central Africa.

One must point out at the start that the characteristic depigmentation properly belonging to onchocerciasis,showing itself in the pretibial and crural skin and over the enlarged glands, is so typical that it should never cause any diagnostic difficulty (Browne, 1959, 2). Also, the lepromatous or tuberculoid lesions presenting in a skin which is otherwise healthy do not lead to confusion. Of course, leprosy and onchodermatitis can co-exist in the same individual, each modifying the cutaneous manifestations of the other. The pigmentary and tegumentary changes associated with a pruritis of long duration and of onchocercal origin can quite often simulate one of the clinical forms of leprosy.

All the cases considered in this study came from the Equatorial forest of the Province Orientale where the leprosy incidence is very high. A total of 5,349 cases in a population of 45,035 persons, or $11.88 \%$, were under treatment in 1957, without taking account of cases of spontaneous regression, nor of those in whom the disease had been arrested. Cases under treatment were classified as follows:

$\begin{array}{lcc}\text { Type of Leprosy } & \text { Total Number of Cases } & \text { Percentage } \\ \text { Tuberculoid } & 3,889 & 72.71 \\ \text { Indeterminate } & 187 & 3.49 \\ \text { Lepromatous } & 1,104 & 20.64 \\ \text { Dimorphous } & 169 & 3.16 \\ & 5,349 & 100.00\end{array}$

To make a diagnosis of leprosy, everybody is examined unclothed in the oblique light of the sun (in order to reveal early lepromatous lesions); smears for microscopic examination are taken from the non-tuberculoid cases by the scraped incision method, using six body sites (lobe of the ear, forehead, cheek, active border of a lesion, apparently normal skin, nasal mucosa); after staining by the method of Ziehl-Neelsen, a count is made of the acid-fast bacilli following the method of Dharmendra (Cochrane, 1952, Browne, 1955).

In this same district, the percentage of cases attacked by onchocerciasis was similarly very high; in some places all the adults were affected. In case recording of onchocerciasis, each patient is given a complete examination, making special note of the presence of nodules; in general, one takes two small skin snips from the neighbourhood of the right iliac crest, which are examined at once in physiological saline, using a weak magnification of the microscope. 
Thanks to this examination the distribution of the cases of onchocerciasis can be determined, always taking account of the fact that the percentage would in reality be higher if one had taken several skin snips. Nevertheless, previous studies have shown that onchocerciasis is usually made evident by these methods, and the infection was massive in comparison with the European findings. In the same population of 45,035 persons there were 7,781 with onchocerciasis, or $17.3 \%$.

The picture of the skin lesions in onchocerciasis is that long known under the name "la gale filarienne", or filarial itch; this name comprises all the modifications which arise from a non-specific pruritis. These modifications may be considered according to the following clinical groupings, which correspond approximately to the degrees of morbidity and in general the duration of the disease.

1. First stage; there is an urticarial reaction with a sub-acute papular, or vesicular, dermatitis; the lesions appear by preference in the scapular and lumbar regions, and in the buttocks and thighs. Without taking account of the secondary lesions due to scratching, the manifestations of this stage are seen as circular or oval plaques on an oedematous skin; the pores are more prominent than usual; the plaque is surrounded by a narrow, circinate, hypopigmented zone.

2. Second stage; there is xerodermia and keratodermia, bound up with a long-standing pruritis which results in constant scratching and rubbing. At times there is a secondary pyodermatitis, and at others super-infection of the impetigo type. The skin of the back forms folds.

3. Third stage; the thickening of the skin disappears, yielding to an atrophy which becomes more and more marked in the skin and its appendages, and a progressive fibrosis of the dermis.

These three stages are represented in the district in the following proportions:

\begin{tabular}{lcrrrrr} 
& Total Number of those & \multicolumn{5}{c}{ Onchodermatitic Stage } \\
& $\begin{array}{l}\text { Affected by Onchocerciasis } \\
\text { Men }\end{array}$ & 1 & 2 & \multicolumn{1}{c}{ Total } & $\%$ \\
Women & 4,633 & 616 & 566 & 231 & 1,413 & 30.5 \\
Children & 2,597 & 200 & 165 & 37 & 402 & 15.5 \\
& 551 & 21 & 9 & 0 & 30 & 5.4 \\
& 7,781 & 837 & 740 & 268 & 1,845 & 23.7
\end{tabular}

Since the areas which have the highest percentage of leprosy are also those where the incidence of onchocerciasis is highest, it is evident that many people will have the two diseases; furthermore, the percentage of onchocerciasis patients having filarial itch, especially in the advanced stages, is very much higher in the villages where most of the inhabitants suffer from onchocerciasis.

Also there are many other diseases which can modify the appear- 
ance of the skin; there are scars of various kinds, including those due to yaws, to true itch, to wounds, to mycoses; the effects of the sun or of heat (erythema ab igne, ephilides ab igne); avitaminoses; changes due to senility (hyperpigmented macules, telangiectases, papillomatous neoplasms, skin atrophy).

Sometimes, the picture of the various lesions on a relatively large expanse of skin gives the impression of a non-specific traumatic chloasma; but considering especially the hypopigmented areas, one must admit that their form and arrangement scarcely resemble the lesions of this nature.

In order better to understand and recognise and so avoid diagnostic traps, we may arrange these cases in the following groups: 1. Simulating Lepromatous Leprosy

In this series there were 19 cases thought at first to be lepromatous and 74 cases which would have received this diagnosis by unwary workers. All these cases have never had leprosy; the lesions were all onchocercal.

How then had they come to be diagnosed as lepromatous leprosy? It was because of the presence of numerous macules, small and hypopigmented and vague, which were scattered symmetrically over the regions of the scapulae, loins, buttocks, and often of the thighs, all simulating very closely lepromatous leprosy.

On the other hand, the macules are generally found on a fairly restricted area of the body which is quite well delimited; these were really too symmetrically disposed and the other parts of the body remained completely free. Repeated microscopic examination did not show any acid-fast bacilli in these cases, which should have had them in abundance if they were really leprosy. Amongst those who were given sulphone therapy while they awaited the results of bacterial examinations there was no clinical improvement.

The examinations of dermal fluid, or of skin snips in all cases, except those where there was excessive atrophy of the skin, showed numerous microfilariae of $O$. volvulus.

These hypopigmented macules probably follow different lines of development. Some begin as urticarial lesions during the early period of sensitization to the filarial or microfilarial toxins. Localised oedema can last some months or disappear slowly, leaving behind certain vague traces of hypopigmentation, or an area of "pig-skin" surrounded by a narrow hypopigmented band. The others seem to be places of local atrophy and of cicatrisation, superimposed on a generally hypertrophic and wrinkled skin.

In severe onchodermatitis, there may be small elevations in the skin which are smooth and normally pigmented and which strongly resemble small lepromas. These little swellings are of two different types; some are multiple small lipomas (Kirk, 1947) and others are true onchocercal cysts, either typically firm in consistency and con- 
taining very numerous microfilariae which have not yet arrived at maturity, or containing degenerated microfilariae and filled with a yellowish liquid, thick and sticky. Careful palpation easily distinguishes them from lepromatous nodules.

The final cicatricial stage of onchodermatitis is characterised by smooth, hypopigmented rhomboidal plaques and by a trophic and inelastic skin (sometimes even with small senile hyperpigmented macules). This should never be confused with the manifestations of leprosy.

2. Tuherculoid Leprosy Simulating Lepromatous Leprosy'

This group contains cases of tuberculoid leprosy in process of regression which, because the lesion is present in a skin modified by chronic onchodermatitis, comes to resemble a lepromatous lesion. There were 18 cases in this group. If there had not been careful clinical observation throughout the years, the existence of these cases would not have been suspected. The difficulty of diagnosis depends on the chance that the tuberculoid lesion occurs in a region of the body which is a site of predeliction for onchodermatitis, namely the back, the buttocks and the thighs.

These macules are in general completely flat, hypopigmented and multiple; their margins are ill-defined, unlike tuberculoid lesions in process of cure in a skin otherwise normal. It seems that whenever the skin is hyperkeratotic following onchodermatitis any pigmentary changes in the tuberculoid lesions in process of cure tend to become more diffuse.

The members of this group were mostly adults suffering from advanced onchodermatitis.

Acid-fast bacilli are never recovered in the tissues, it follows that the lesions cannot be lepromatous even in regression.

Whether apparent cure is due to treatment or comes without any treatment, the final aspect is the same, namely of ill-defined macules completely flat in a thick onchodermatitic skin. (There are other cases, quite numerous in fact, where tuberculoid lesions in an onchodermatitic skin are not modified in the manner indicated above.)

\section{Onchodermatitis Simulating Tuberculoid Leprosy}

This group contains three cases where the onchodermatitis depigmentation resembled tuberculoid lesions without there ever having been leprosy in that part of the body.

In each case there were some discrete and well delimited macules in the lumbar region; their borders resembled the typical tuberculoid lesions, but the uniformity of the hypopigmentation in the centre and the atrophy of the skin inclined one to think of onchodermatitis rather than leprosy.

\section{Lepromatous Leprosy Simulating Onchodermatitis}

This group contains three cases where the hypochromic lesions 
strongly resembled onchodermatitis, but really were early lepromatous macules. There were numerous microfilariae in the skin and the general appearance of the skin was typical of onchodermatitis. But routine examination revealed very numerous acid-fast bacilli, isolated and in globi. The therapeutic response to the sulphones left no doubt as to the diagnosis; the hypochromic macules disappeared but the onchodermatitis remains.

\section{Histopathology}

It is unnecessary to review the histological findings which have been known for some time but the special picture may be described as follows:

Examinations of numerous sections leave the impression that there is some localised disturbance in groups of melanocytes without any indication of its cause. Sometimes the melanocytes in the neighbourhood of the microfilariae are flattened or atrophic and their granules are arranged abnormally and divergent types exist.

Later, there begins a progressive fibrosis of the skin, producing an atrophy of the cells of the epidermis, a flattening of the papillae, and the disappearance of pigment of the cells of the basal layer.

The infiltration into the skin is composed at first of histiocytes, eosinophils, and monocytes, and lateralmost exclusively of monocytes, surrounded by fibrous tissue where occasional microfilariae are found.

\section{Summary}

Basing his conclusions on the study of 131 cases in the Oriental Province of the Belgian Congo, the author draws attention to the real and practical diagnostic difficulties that arise in those districts where both leprosy and onchocerciasis are highly endemic.

The depigmentary changes in the skin in some cases of onchodermatitis ("craw-craw") may be confused with lepromatous macules, or even with tuberculoid lesions that are healing. The cutaneous manifestations of the two polar forms of leprosy may be modified by reason of the fact that they are grafted on to a skin that is the seat of onchodermatitis.

Careful clinical examinations, with an accurate history, must always be completed in these difficult cases by microscopical examinations of the dermal fluid to discover microfilariae and $M$. leprae and, where necessary, by histological examination of biopsy material.

\section{References}

Browne, S. G. (1955) Internat. J. Lep., 23, 284.

"Leprosy in Theory and Practice", edited By Cochrane, R. G. John Wright \& Sons Ltd. (Bristol 1959) 152.

Cochrane, R. G. (1952) Leprosy Review, 23, 135.

Dubois, A. (1917) Bull. Soc. Path. exot., 10, 365.

KIRK, R. (1947) Ann. Trop. Med. Parasit., 41, 357.

O'Neill, J. Lancet, 1875, 265.

Ouzilleau, F., Baigret, J., and Lefrou (1921) Bull. Soc. Path. exot., 14, 717

Rodhain, J. (1915) Bull. Soc. Path. exot., 8, 740.

Tisseuil, J. (1949) Bull. Soc. Path. exot., 42, 539.

Van Der Berghe, L. (1941) Ann. Soc. Belge. Med. Trop., 21, 63, 167 and 261. 